who support the personal use of heroin being made legal (8.1 per cent) has not changed since 1995 .

\section{DISCUSSION}

The use of heroin is not widespread in NSW, with less than one per cent of the population reporting recent use. There is also no indication from the NDSHS that recent heroin use is increasing. Indeed, the previously reported high rates of recent heroin use among males aged 20-29 and females aged 14-19 have declined notably.

There are, however, a number of limitations with the NDSHS that must be taken into consideration when interpreting the results presented here. First, the overall survey response rate was 56 per cent, which may have resulted in some selection bias. If illicit drug users were less likely to participate then drug use would be underestimated. Other potential limitations include: a small NSW sample size for low-prevalence behaviours; the exclusion of non-private dwellings, institutional settings (including prisons) and homeless people from the sample; and potential reporting and recall bias when recounting illicit drug use. These limitations have been discussed in more detail elsewhere. ${ }^{1,2}$

The increased public and government concern about heroin as a 'drug problem' reflects increased coverage and discussion around both patterns in heroin use and trends in heroin-related overdoses. Accordingly, the 1999 NSW Drug Summit primarily concentrated on illicit drugs, especially heroin, in developing new responses to problematic drug use. In response, the NSW Government is providing a significant expansion of-and enhancement to-drug treatment services, supported by innovative approaches to drug education and prevention. The NSW Department of Health is presently developing a five-year Drug Treatment Services Plan that will outline statewide goals and priorities for service delivery and expansion.
Note: With regard to type and source of heroin used, and mode and place of administration, there are differences in the calculation of the percentages in this report. All data relating only to NSW excludes non-responders from the denominator, while data presenting interstate or capital city comparisons includes non-responders. These data were derived from that provided by the AIHW.

\section{ACKNOWLEDGMENTS}

This series of articles is a joint enterprise of the Australian Institute of Health and Welfare and the NSW Department of Health. The assistance of the following groups is particularly appreciated: the Department of Health and Aged Care Policy Reference Group, and the Survey Technical Advisory Committee.

\section{REFERENCES}

1. Thackway S, Poder A, and Summerill A. Licit and illicit drug use in NSW. NSW Public Health Bulletin, December 1999.

2. Australian Institute of Health and Welfare. 1998 National Drug Strategy Household Survey: First Results. AIHW cat. no. PHE 15. Canberra: AIHW Drug Statistics Series, 1999.

3. New South Wales Government. NSW Drug Summit: Government Plan of Action. Sydney, NSW Government, 1999.

4. Epidemiology and Surveillance Branch, NSW Department of Health. ABS Deaths Data. Sydney: Health Outcomes Information Statistical Toolkit, NSW Department of Health, 1999. 䨛

Further information on national statistics, response
rates, estimation procedures, reliability of
estimates, and comparability to the 1995 survey,
are available in the AlHW Drug Statistics Series (1),
which can be accessed from the AlHW Web site at
www.aihw.gov.au.

\title{
THE ILLICIT DRUGS REPORTING SYSTEM
}

\section{Rebecca McKetin}

National Co-ordinator

Illicit Drug Reporting System

National Drug and Alcohol Research Centre

University of New South Wales

This article outlines the purpose and methodology of the Illicit Drug Reporting System (IDRS), an initiative funded by the Commonwealth Department of Health and Aged Care; and presents major trends identified by the IDRS in 1999.

\section{PURPOSE AND METHODOLOGY OF THE ILLICIT DRUG REPORTING SYSTEM}

The IDRS aims to monitor data on the use of opiates, cocaine, amphetamine and cannabis. It is intended to act as a strategic warning system by identifying problematic drug trends that require further investigation. As such, the IDRS aims to be sensitive to emerging drug trends of national significance. Findings from the IDRS are disseminated to the Commonwealth Department of Health and Aged Care, the Inter-Governmental Committee on 


\section{FIGURE 1}

OVERDOSE FATALITIES AMONG THOSE AGED 15-44 YEARS IN NSW, RATE PER MILLION OF POPULATION, 1991-1998

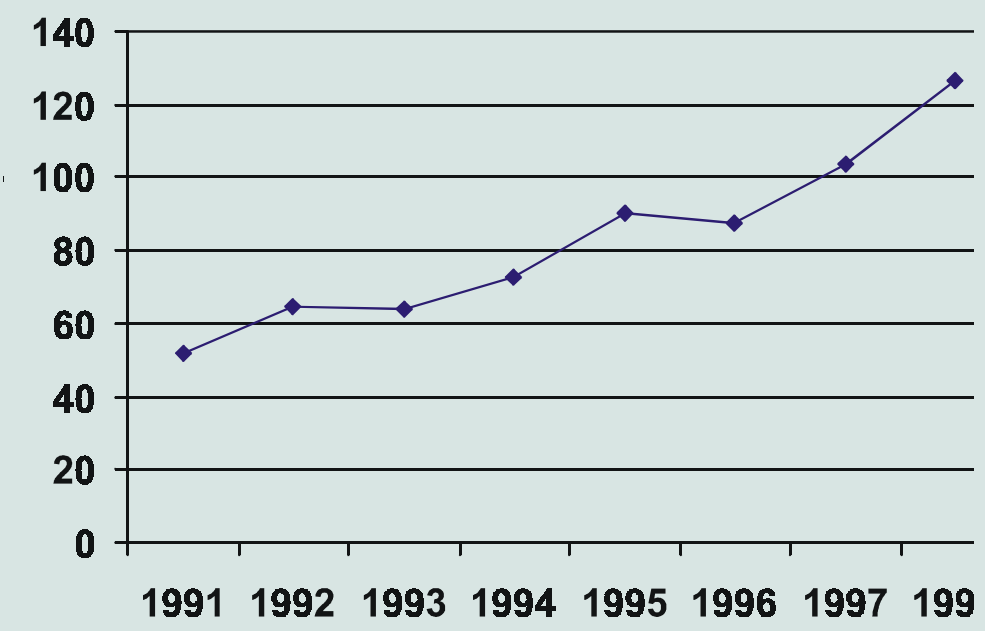

Drugs, the Australian National Council on Drugs, and the Ministerial Council on Drug Strategy.

The IDRS has been operational in NSW since 1996, where it has proved able to detect new and problematic drug trends. Drug trends are detected by examining information from three sources:

- $\quad$ survey of injecting drug users (IDUs)

- survey of key informants

- other indicators,

which are described below. These three data sources both complement and supplement each other, describing both qualitative and quantitative aspects of drug trends, and providing evidence of convergent validity.

\section{Survey of Injecting Drug Users}

The IDU survey consists of annual face-to-face interviews with at least 150 IDUs recruited from sentinel drug-using regions in Sydney. A standardised and structured interview schedule is administered to participants, and includes sections on:

- demographics

- use of drugs

- price of drugs

- purity and availability of drugs

- drug-related crime
- risk-taking behaviour

- health and general drug trends.

Previous IDRS research has found that IDUs are a good group for detecting trends in illicit drug use, due to their high exposure to many types of illicit drugs and their first-hand knowledge of drug price and availability.

\section{Survey of key informants}

At least 30 key informants, who work in the field of illicit drugs, are interviewed each year. These key informants have at least weekly contact with users of illicit drugs, or contact with at least 10 different users of illicit drugs, in the past six months. Key informants include:

- health professionals

- Needle and Syringe Program (NSP) workers and outreach workers

- law enforcement officers

- researchers.

Key informants are interviewed, usually over the phone, using a semi-structured interview schedule which parallels the interview schedule of the IDU survey described above. Interviews of key informants have been found to provide good qualitative data on the context of drug use and healthrelated issues, such as presentations for treatment.

\section{Other indicators}

To complement and validate data collected from both the 
IDU and key informant surveys, a range of secondary data sources are examined. These include:

- health data

- survey data

- law enforcement data.

Most indicator data includes at least 50 cases annually, and covers several drug classes. Examples of data sources include:

- telephone advisory data

- purity of drug seizures made by the Australian Federal Police

- population surveys

- NSP client data

- toxicology data on drug-related deaths and urine of methadone patients

- drug arrest data.

The above IDRS methodology was initially trialled in 1996 in NSW, in accordance with recommendations made by Wardlaw Consulting in $1994 .{ }^{1}$ Data collection was then expanded to South Australia and Victoria in 1997-98. In 1999 the IDRS became a national project, with an abbreviated form of the methodology being conducted in the remaining States and Territories. This methodology consisted of a survey of key informants and collection of other indicator data.

\section{DRUG TRENDS IN NSW, 1999}

Several major trends were detected in NSW by the IDRS during 1999. These included changes in the demographics of injecting drug users, and trends in heroin use. These are summarised below.

\section{Trends in the demographics of IDU}

The frequency of injecting among the NSW IDU sample had increased substantially since 1997. In 1997, 26 per cent of subjects reported injecting more than once a day, compared to 70 per cent in 1999. Importantly, younger IDUs ( $<=25$ years) reported more frequent injections than older IDUs, with 82 per cent of younger IDUs injecting more than once a day in the preceding month, compared to 62 per cent of older IDUs. Also, the average age of the initiating injecting drug user was two years younger in 1999 than it was in 1997 (17.1 years in 1999 versus 19.0 years in 1997). These younger IDUs were also significantly more likely to report having injected heroin as their first drug in 1999 (76 per cent in 1999 versus 49 per cent in 1997), whereas initial use of amphetamine was more likely among older subjects in 1997 (47 per cent in 1999 versus 21 per cent in 1997). Overall, there appears to have been a major shift in initial drug use patterns among younger IDUs, who initiated injection at a younger age and, overwhelmingly, commenced their IDU careers with heroin.

\section{Trends in heroin use}

Although heroin use remains relatively low among the general population, ${ }^{2}$ its use among IDUs appears to have increased dramatically over the last four to five years. According to the Australian Needle and Syringe Program Survey, heroin is the most popular injected drug among IDUs in NSW. The prevalence of heroin, as the last drug injected, has increased from 31 per cent to 48 per cent since 1995. Consistent with the survey findings, many participants in the IDRS IDU survey and key informant survey noted an increase in the number of heroin users, especially an increase in the number of young heroin users and more 'mainstream' people using heroin. There was also a perception among key informants and IDUs that heroin users were using more heroin in a day than previously observed. This observation is consistent with findings mentioned above, that there has been a dramatic increase in the frequency of injection among the IDUs surveyed by the IDRS.

Local indicator data collected by the IDRS was consistent with an increase in the prevalence of heroin use. Trends in specific indicators of heroin use are summarised below.

- The number of criminal incidents relating to narcotic use and/or possession in NSW increased from 1,541 in 1996 to 3,020 in 1998.

- The number of methadone clients in NSW, on the 30th of June each year, has increased steadily throughout the 1990s: from 5,009 in 1990 to 12,549 in 1999.

- The number of heroin-related telephone inquiries to the Alcohol and Drug Information Service in NSW increased from 3,670 in the 1996-97 financial year to 6,763 in 1998-99. There are now more inquiries relating to heroin than to any other illicit drug.

- The number of confirmed overdose fatalities has risen from around 100-200 per year between 1990 and 1993 to 358 in 1998 , and account for nearly half the overdose fatalities in Australia. Overdose rates for 1991 to 1998 are shown in Figure 1. Local indicator data on suspected drug-related deaths also suggests a substantial increase in the number of overdoses occurring in regional NSW.

All of the IDRS indicators point toward an increase in heroin use over the last few years, characterised by younger users of heroin, more 'mainstream' users of heroin, and an increase in the frequency of heroin use among regular users. There was a corresponding increase in heroin-related problems, most notably overdose fatalities. New South Wales has the highest rate of overdose fatalities in 
Australia, accounting for nearly half the overdose fatalities in the country.

\section{CONCLUSION}

The frequency of heroin use among IDUs has risen substantially over the last few years, and all indicators of heroin use suggest that there has been an increase in the number of heroin users in NSW. Harm to public health associated with injecting heroin use continues to be prominent, particularly overdose fatalities.

\section{REFERENCES}

1. Wardlaw, G. Illicit Drug Reporting System. Consultant's Report to the Commonwealth. Canberra: Department of Human Services and Health and Wardlaw Consulting Pty Ltd, 1994.

2. Australian Institute of Health and Welfare. 1998 National Drug Strategy Household Survey: First Results. Canberra: Australian Institute of Health and Welfare, 1999. it

The following IDRS reports are available through the National Drug and Alcohol Research Centre by telephone on (02) 9398 9333; by fax on (02) 93997143 :

- Drug Trends Bulletin: a free quarterly report from the IDRS that contains an update on recent drug trend information;

- State reports: annual reports that contain the findings of the IDRS from each state and territory $(\$ 10$ each);

- National report: an overview of Australian drug trends $(\$ 10)$.

For a summary of the latest drug trends visit the IDRS Web site at: www.med.unsw.edu.au/ndarc/idrs.

\section{NSW DRUG SUMMIT UPDATE}

Kee Foong and Jennifer Gray

NSW Drug Programs Bureau

NSW Department of Health

In May 1999, the NSW Drug Summit was held at the NSW Parliament. For one week members of State Parliament, drug experts, parents, former drug users, and community leaders, met to decide how best to deal with the drug problem in NSW. The summit endorsed a number of resolutions, the majority of which were accepted by the NSW Government. A Government Plan of Action (GPA) was developed, and this article describes initiatives developed to implement this plan.

The GPA describes a comprehensive, 'whole of government' and 'whole of community' approach to the prevention and management of illicit drug misuse. Over $\$ 176$ million has been provided by the State Government to implement a range of new initiatives over the next four years. This has been the most significant enhancement in funding to drug and alcohol services since the National Drug Strategy in 1985. To coordinate this significant investment the State Government has established the Office of Drug Policy within the NSW Cabinet Office. An expert advisory committee, reporting to the Premier, has also been established and is chaired by Professor Ian Webster.

\section{IMPLEMENTATION}

NSW Health has received significant enhancements. The new monies have been allocated to significantly enhance service provision; and to ensure that a system built on quality, equity and fairer access is in place. The Drug Programs Bureau (formerly the Drug and Alcohol Directorate) within the Public Health Division, and the Area Health Services (AHS), have primary responsibility for more than 100 projects resulting from the GPA. 